\title{
COMMUNICATIONS
}

\section{Design and miniaturization of dual-band Wilkinson power dividers}

\author{
Vaidotas Barzdenas ${ }^{1,2 *}$, Aleksandr Vasjanov ${ }^{1,2}$, \\ Gediminas Grazulevicius ${ }^{1}$, Andzej Borel ${ }^{1,2}$
}

\begin{abstract}
In this paper, four differently shaped Wilkinson power dividers are presented by selecting the same physical length of twosection transmission lines, dual arbitrary frequency band Wilkinson power dividers can be achieved. The 2.4 GHz (WLAN) and 5.9 GHz (DSRC IEEE 802.11p) frequency bands are selected to complement the future development of multi-band, multi-standard transceivers. To improve physical separation and electrical isolation between the two output ports a parallel RLC circuit is employed. For verification, the simulated and measured performance results of dual-band Wilkinson power dividers implemented on the Rogers 4003C laminate are presented. The measurement results for the fabricated Wilkinson power dividers were in good agreement with theoretical simulation results and show dual-band characteristics.
\end{abstract}

K e y w o r d s: dual-band, DSRC, RF/microwave circuit, Rogers 4003C, Wilkinson power divider (WPD)

\section{Introduction}

Wilkinson power dividers/combiners (WPD) are one of the key passive microwave components of modern microwave and RF communication systems. They are widely used for power division or combination in different microwave circuits such as power amplifiers, antenna feeding networks, I/Q vector modulators, demodulators, mixers, frequency multipliers, etc. WPD became popular due to their planar structure simplicity, good input and output port matching, and isolation characteristics, but conventional distributed Wilkinson power dividers require a large area at printed circuit board (PCB). For this reason, miniaturization of power dividers has become an attractive topic for microwave researchers and designers. Thereby, various structures have been proposed over the years offering dual- or multi-band WPD with improved performances: low insertion loss, improved isolation and matching, wide band-ratio, small board size [18], but all of these designs do not include the $5.9 \mathrm{GHz}$ band. Therefore, in this paper, we present four differently shaped WPD with the same physical length of twosection transmission lines for $2.4 \mathrm{GHz}$ and $5.9 \mathrm{GHz}$ frequency bands, which are widely used in wireless local area network (WLAN) and dedicated short-range communications (DSRC IEEE 802.11p) applications.

We have design the four different shaped dual-band Wilkinson power dividers. The fabricated prototypes were approved by a comparison of the simulation and measured results. The appendicles contain results describing two of the four structures presented in this paper.

\section{Design, equations and miniaturization}

As mentioned, this work focuses on the design and implementation of a dual-band WPD with the parallel RLC circuit. The circuit schematic of this power divider is shown in Fig. 1 and consists of two pairs of transmission lines with same physical lengths $l_{1}, l_{2}$ and different characteristic impedances $Z_{1}, Z_{2}$, where $\left(l_{1}, Z_{1}\right)$ and $\left(l_{2}, Z_{2}\right)$ correspond to operating frequencies $f_{1}$ and $f_{2}$, respectively. The parallel connection of the resistor $(R)$, the capacitor $(C)$, and the inductor $(L)$, which shunt the two output ports, are used to increase isolation between the outputs. $Z_{0}$ is the port characteristic impedance.

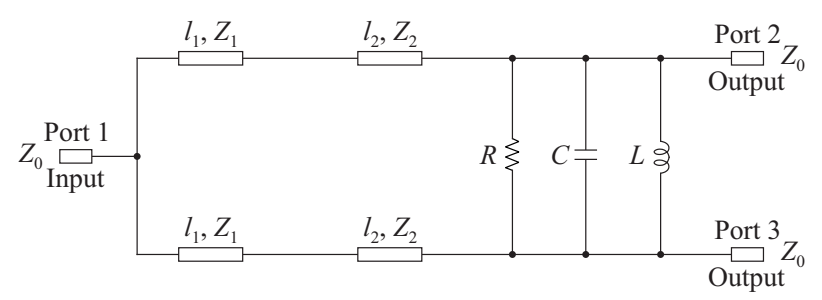

Fig. 1. Dual-band WPD with the parallel $R C L$ isolation circuit

Methods for calculating parameters of such a power divider structure are described in various references [911]. According to [10], the physical length of two-section transmission lines can be obtained as

$$
l_{1}=l_{2}=\frac{n \pi}{\beta_{1}+\beta_{2}},
$$

where $n$ is a positive integer and, in this case, is equal to 1 , because the ratio of $f_{2} / f_{1}$ is less than 3 . Here, $\beta$

\footnotetext{
${ }^{1}$ Department of Computer Science and Communications Technologies, Vilnius Gediminas Technical University, 03227 Vilnius, Lithuania, Micro and Nanoelectronics Systems Design and Research Laboratory, Vilnius Gediminas Technical University, 10223 Vilnius, Lithuania, *Corresponding author vaidotas.barzdenas@vgtu.lt
} 


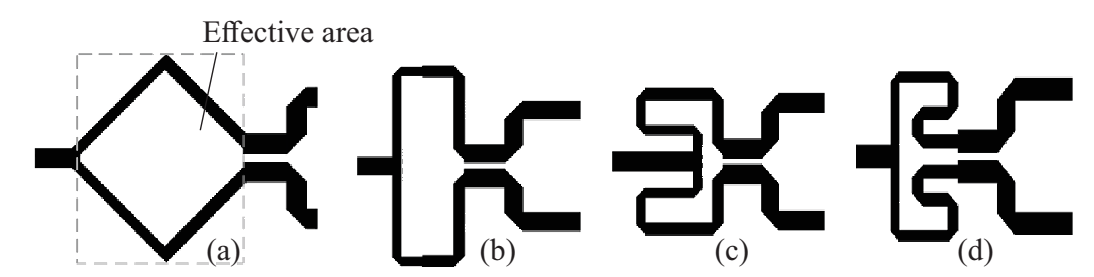

Fig. 2. Dual-band WPD configurations: (a) - rhombus type, (b) - rectangle type, (c) - $l_{1}$-folded type, and (d) - $l_{2}$-folded type

Table 1. Design parameters of the dual-band WPD

$$
\begin{aligned}
& p=\tan \left(\beta_{1} l_{1}\right), \\
& q=\tan \left(\beta_{2} l_{1}\right) .
\end{aligned}
$$

\begin{tabular}{lc} 
Parameter & Value \\
\hline$l_{1}=l_{2}$ & $11.1 \mathrm{~mm}$ \\
$W_{0}$ & $1.8 \mathrm{~mm}$ \\
$W_{1}$ & $0.85 \mathrm{~mm}$ \\
$W_{2}$ & $1.1 \mathrm{~mm}$ \\
$Z_{0}$ & $50 \Omega$ \\
$Z_{1}$ & $75.4 \Omega$ \\
$Z_{2}$ & $66.3 \Omega$ \\
$R$ & $100 \Omega$ \\
$C$ & $0.1 \mathrm{pF}$ \\
$L$ & $17.1 \mathrm{nH}$ \\
\hline
\end{tabular}

Calculations were performed based on the above mentioned equations and the fact that the dual-band Wilkinson power divider will have carrier frequencies of $f_{1}=$ $2.4 \mathrm{GHz}$ and $f_{2}=5.9 \mathrm{GHz}$, and will be implemented on a $0.813 \mathrm{~mm}$ thick Rogers $4003 \mathrm{C}$ laminate. The latter laminate was considered to have a relative permittivity of 3.55 , conductor cladding $17 \mu \mathrm{m}$ in thickness on both sides, and a dissipation factor $(\tan \delta)$ of 0.0021 at $2.5 \mathrm{GHz} / 23^{\circ} \mathrm{C}$, [12]. Summary of the proposed dual-band WPD segment values is tabulated in Tab. 1. Considering the fact, that the electrical lengths of $l_{1}$ and $l_{2}$ should be left constant, the only way of reducing the overall area is to fold the segments in such a way, which introduces is the propagation constant for $f_{1}$ and $f_{2}$ frequencies, respectively.

The characteristic impedances of the transmission lines $Z_{2}$ and $Z_{1}$, are

$$
\begin{gathered}
Z_{2}=Z_{0} \sqrt{\frac{1}{2 \alpha}+\sqrt{\frac{1}{4 \alpha^{2}+2}}}, \\
Z_{1}=\frac{2 Z_{0}^{2}}{Z_{2}},
\end{gathered}
$$

where $Z_{0}$ is the ports characteristic impedance $(50 \Omega)$, and parameter is

$$
\alpha=\tan ^{2}\left(\beta_{1} l_{1}\right)
$$

The values of the isolation resistor, capacitor and inductor can be express by

$$
\begin{gathered}
R=2 Z_{0}, \\
C=\frac{B / \omega_{1}-A / \omega_{2}}{2 \omega_{2} / \omega_{1}-2 \omega_{1} / \omega_{2}}, \\
L=\frac{2 \omega_{2} / \omega_{1}-2 \omega_{1} / \omega_{2}}{B \omega_{1}-A \omega_{2}},
\end{gathered}
$$

where $\omega$ is the radian frequency for $f_{1}$ and $f_{2}$ frequencies, respectively, and $A, B, p$ and $q$ parameters can be expressed by the following equations:

$$
\begin{aligned}
& A=\frac{Z_{2}-Z_{1} p^{2}}{Z_{2} p\left(Z_{1}+Z_{2}\right)}, \\
& B=\frac{Z_{2}-Z_{1} q^{2}}{Z_{2} q\left(Z_{1}+Z_{2}\right)},
\end{aligned}
$$

the least amount of stray capacitance between the transmission lines, but still reduces the overall area. Thus, based on the parameters in Tab. 1, four differently shaped WPDs are presented and discussed. All the latter configurations are shown in Fig. 2. The first WPD shown in Fig. 2 (a) is the rhombus type, and the entire structure occupies an area of $3 \mathrm{~cm}^{2}$ on the PCB. The effective area is calculated by considering only the area occupied by two-section transmission lines as highlighted by the dotted line in Fig. 2(a). The other three structures were designed to reduce this area while maintaining the calculated parameters listed in Tab. 1. The second structure is the rectangle type and shown in Fig. 2(b), covering an area of $1.4 \mathrm{~cm}^{2}$. The remaining two reduced-size configurations are shown in Fig. 2(c) and Fig. 2(d) and are named $l_{1}$-folded and $l_{2}$-folded, respectively. The latter names are assigned based on which power divider transmission line pair ( $l_{1}$ at the input or $l_{2}$ at the output) is symmetrically folded. The resulting areas are $1.2 \mathrm{~cm}^{2}$ and about $1 \mathrm{~cm}^{2}$, respectively. Thus, compared to the rhombus type configuration, the area of the latter two power dividers is reduced about three times.

\section{Simulation and measurement results discussion}

To verify the above mentioned power divider structures, the four different design examples are implemented on Rogers 4003C laminate. Figure 3 presents a photograph of the fabricated prototypes alongside a metric ruler. The impedances at each port have been designed to be nominal $50 \Omega$. The isolation resistor, capacitor and inductor are surface-mount 0402-size components and have the values of $100 \Omega$ (1\% tolerance), $0.1 \mathrm{pF}( \pm 0.1 \mathrm{pF})$, 


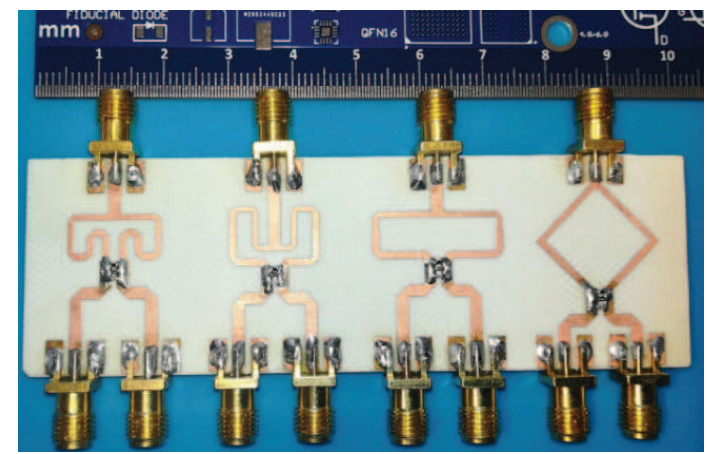

Fig. 3. Photograph of the fabricated dual-band WPD structures

and $17 \mathrm{nH}$ (1\% tolerance), respectively. The simulated and measured results are obtained using the Keysight ADS2017 software package and Rohde \& Schwarz ZVB8 vector network analyzer (VNA), respectively. The VNA has been calibrated accounting for the length of the cable and the reference plane has been moved right after the connector, as the distance from the connector to the PCB microstrip and the loss in the connector are known [13].

The designed rhombus type WPD is set as the base structure regarding the S-parameters. The folded structures are compared to the latter to maintain the performance while reducing the effective area, marked in Fig. 2 (a). The simulations and measurements have been conducted over the frequency band ranging from $10 \mathrm{MHz}$ to $8 \mathrm{GHz}$. Rectangle type WPD was found to be the best performing structure, thus the measurement and simulation results are presented alongside the base structure in Fig. 4.
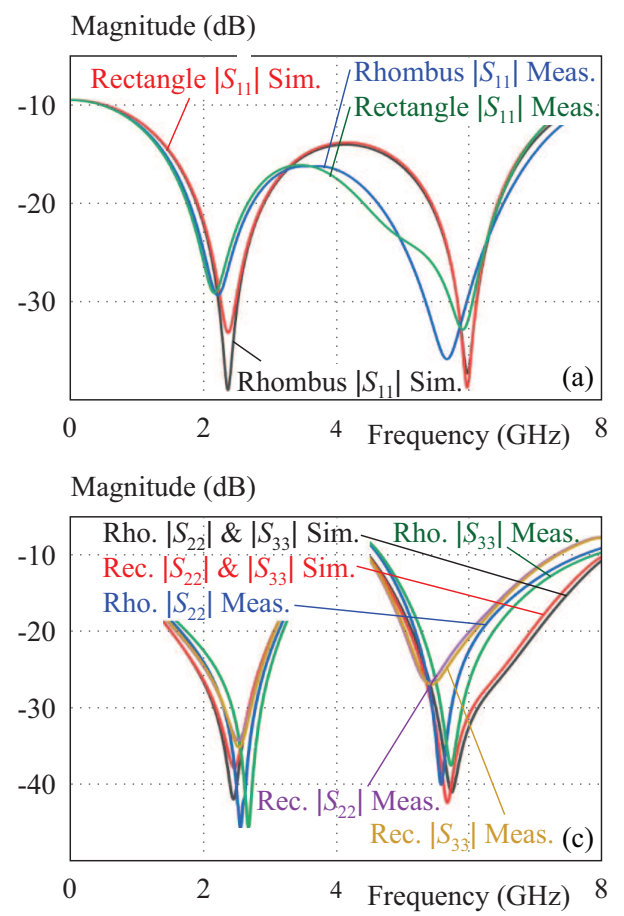

Comparing the rhombus and rectangular WPD structures, the measured return loss $\left(S_{11}\right)$ is lower than -26.1 $\mathrm{dB}$ and $-31.8 \mathrm{~dB}$ for $2.4 \mathrm{GHz}$ and $5.9 \mathrm{GHz}$, respectively, as shown in Fig. 4(a). The measured insertion loss $\left(S_{21}\right.$ and $\left(S_{31}\right)$ is in the range of $-3.05 \mathrm{~dB}$ to $-3.3 \mathrm{~dB}$, Fig. 4(b) and is close to the simulated values. The measurement results show and a good output return loss $\left(S_{22}\right.$ and $\left.S_{33}\right)$ which is better than $-21 \mathrm{~dB}$ at both target frequencies, Fig. 4(c). The isolation $\left(S_{23}\right.$ and $\left.S_{32}\right)$ between Port2 and Port3 (Fig. 1) is lower than $-28 \mathrm{~dB}$ at 2.4 $\mathrm{GHz}$, and $-21 \mathrm{~dB}$ at $5.9 \mathrm{GHz}$ as shown in Fig. 4(d). Thus, folding the rhombus type dual-band Wilkinson power divider in a rectangular way ensuring a minimum amount of stray capacitance between the microstrip segments reduces the effective area by $53 \%$ (in the case of this paper from $3 \mathrm{~cm}^{2}$ to $1.4 \mathrm{~cm}^{2}$ ) while maintaining almost identical performance and can lead to more compact multiband, multi-standard transceivers for vehicular communications.

Folding the rectangle WPD structure introduces parasitic stray capacitance between the microstrips, which affects the S-parameter response curves. Even though $l_{1}$ -folded type and $l_{2}$-folded type WPD performed as expected around $2.4 \mathrm{GHz}$ with an $S_{11}$ curve minimum offset to the lower side by around a $100 \mathrm{MHz}$ when compared to the simulation results, the additional stray capacitance greatly affected the performance around $5.9 \mathrm{GHz}$. The $S_{11}$ minimum shifted to the lower frequency range by more than $250 \mathrm{MHz}$ and the minimum $S_{11}$ magnitude degraded by around $15 \mathrm{~dB}$. The return loss $\left(S_{21}\right.$ and $\left.S_{31}\right)$ also increased at $5.9 \mathrm{GHz}$ by more than $0.2 \mathrm{~dB}$. Complete measurement results for all investigated structures are presented in Appendix A.
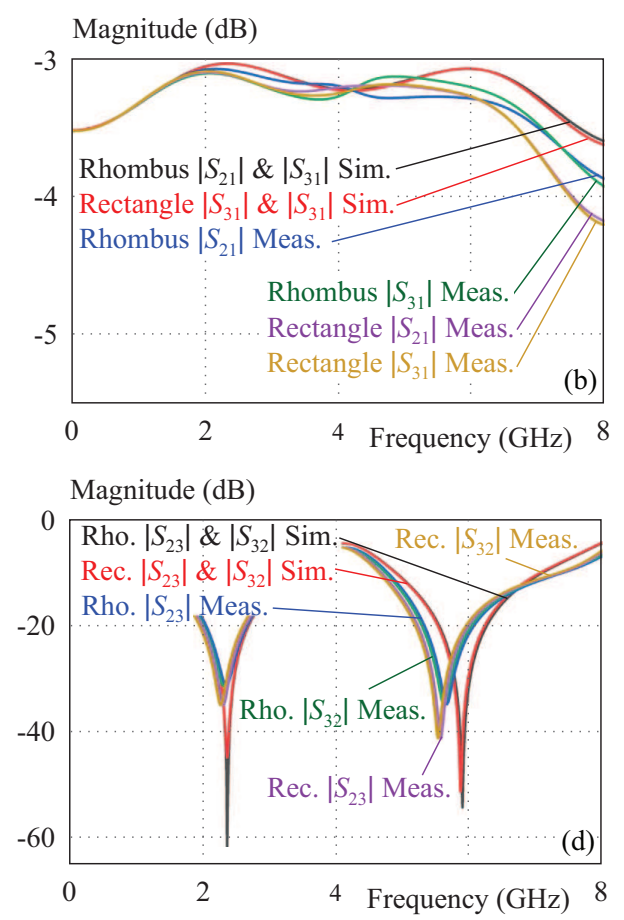

Fig. 4. Rhombus and rectangle type dual-frequency WPD S-parameter simulation and measurement results: (a) - return loss $\left(S_{11}\right)$, (b) - insertion loss $\left(S_{21}, S_{31}\right)$, (c) - output return loss $\left(S_{22}, S_{33}\right)$, and (d) - output port isolation $\left(S_{23}, S_{32}\right)$ 


\section{Appendix A}
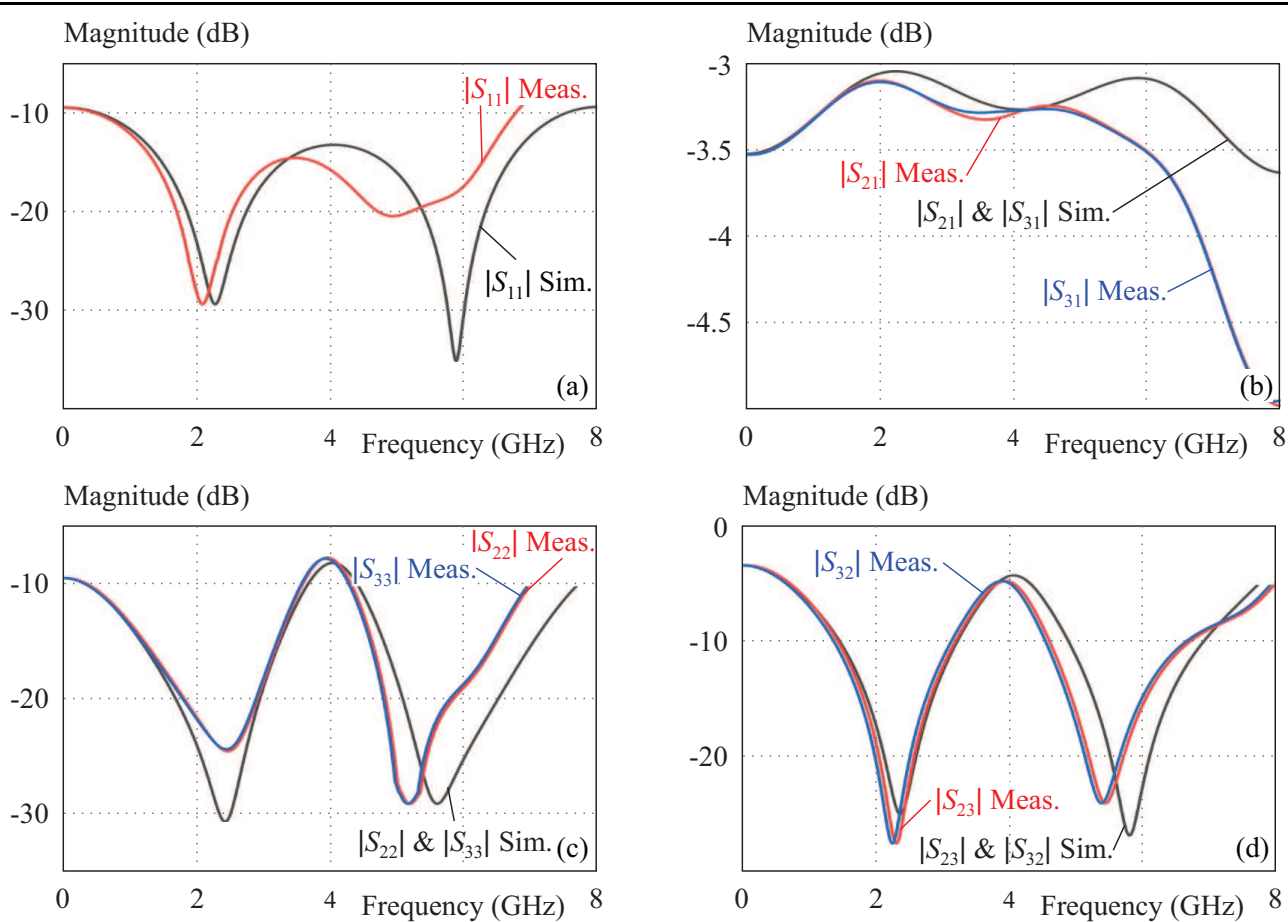

$l_{1}$-folded type dual-frequency Wilkinson power divider S-parameter simulation and measurement results: (a) - return loss $\left(S_{11}\right)$, (b) - insertion loss $\left(S_{21}, S_{31}\right)$, (c) - output return loss $\left(S_{22}, S_{33}\right)$, (d) - output ports isolation $\left(S_{23}, S_{32}\right)$
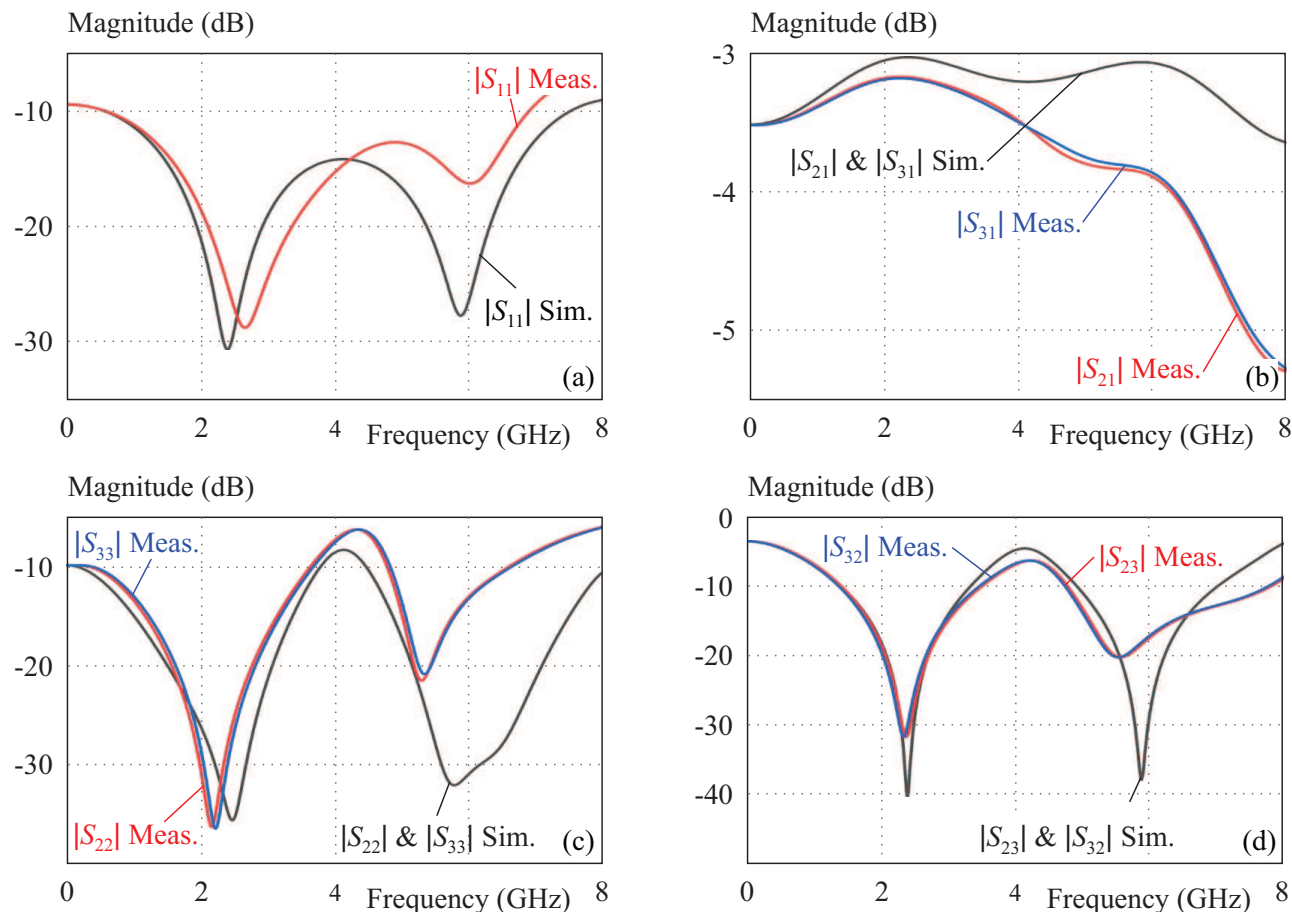

$l_{2}$-folded type dual-frequency Wilkinson power divider S-parameter simulation and measurement results: (a) - return loss $\left(S_{11}\right)$, (b) - insertion loss $\left(S_{21}, S_{31}\right),(\mathrm{c})$ - output return loss $\left(S_{22}, S_{33}\right)$, and (d) - output ports isolation $\left(S_{23}, S_{32}\right)$

\section{Conclusions}

In this paper, four different shaped Wilkinson power dividers with parallel RLC complex isolation components were presented. The size of the investigated power dividers was reduced by folding the same physical lengths two-section transmission lines. The designed rhombus type WPD was set as the performance baseline and only the rectangle type WPD maintained the performance (return and insertion loss as well as port isolation) at 2.4 $\mathrm{GHz}$ and $5.9 \mathrm{GHz}$ arbitrary frequencies as well as reducing the effective area by $53 \%$ (in the case of this paper 
from $3 \mathrm{~cm}^{2}$ to $1.4 \mathrm{~cm}^{2}$ ). All designed WPD structures have been investigated using Keysight ADS2017 software package, fabricated on the Rogers 4003C substrate and measured using Rohde \& Schwarz ZVB8 vector network analyzer. The presented dividers can be easily applied to compact multi-band, multi-standard transceivers for vehicular communications.

\section{Acknowledgements}

The authors would like to thank Rogers Corporation for their generous donation of laminate samples used in the development of the presented prototypes. The authors would also like to thank their colleagues in Micro and Nanoelectronics Systems Design and Research Laboratory for their support while writing this paper.

\section{REFERENCES}

[1] X. Wang, I. Sakagami, Z. Ma, A. Mase, M. Yoshikawa, and M. Ichimura. "Miniaturized dual-band Wilkinson power divider with self-compensation structure", IEEE Transactions on Components, Packaging and Manufacturing Technology, vol. 5, no. 3, pp. 389-397, https://doi.org/10.1109.2015.2402213.

[2] M. Kumar, S. K. Nurul Islam, G. Sen, S. K. Parui, and S. Das. "Miniaturization of Dual-Band Wilkinson Power Divider using Dual Transmission Line", IEEE Applied Electro-magnetics Conference (AEMC), Aurangabad, vol. 19, no. 2017, pp. 1-2, https://doi.org/10.1109.2017.8325701.

[3] H. Zhang, W. Kang, and W. Wu. "Design of a novel compact dual-band Wilkinson power divider with improved isolation", 2016 IEEE International Conference on Microwave and Millimeter Wave Technology (ICMMT), pp. 5-8, https://doi.org/10. 1109.2016.7762505.

[4] M. K. Hedayati, G. Moradi, A. Abdipour, and M. Mosalanejad. "A miniaturized dual-frequency Wilkinson power divider using defected ground structure", 2010 IEEE Asia-Pacific Conference on Applied Electromagnetics (APACE), pp. 9-11, https://doi. org/10.1109.2010 5719770 .

[5] X. Wang, I. Sakagami, and A. Mase. "Generalized, miniaturized, dual-band Wilkinson power divider with series RLC circuit", Asia-Pacific Microwave Conference Proceedings (APMC), pp. 5-8, https://doi.org/10.1109.2013.6695150.

[6] Q. Wang, C. Bao, B. Wu, and X. Wang. "Multiple design approach of dualband Wilkinson power divider with arbitrary transmission line ratio", International Journal of RF and Microwave Computer-Aided Engineering, 2020,30:e22109, https:/ /doi.org/10.1002/mmce.22109.

[7] X. Wang, and I. Sakagami. "Generalized dual-frequency Wilkinson power dividers with a series/parallel RLC circuit", IEEE MTT-S International Microwave Symposium, pp. 5-10, https:// doi.org/10.1109.2011.5972671.

[8] X. Wang, I. Sakagami, K. Takahashi, and S. Okamura. "A Generalized Dual-Band Wilkinson Power Divider With Parallel L, C, and R Components", IEEE Transactions on Microwave Theory and Techniques, vol. 60, no. 4, pp. 952-964, https://doi.org/10.1109.2012.2184298.

[9] C. Monzon. "A small dual-frequency transformer in two sections", IEEE Transactions on Microwave Theory and Techniques, vol. 51, no. 4, pp. 1157-1161, https://doi.org/10.1109. 2003.809675 .
[10] L. Wu, Z. Sun, H. Yilmaz, and M. Berroth, A dual-frequency Wilkinson power divider IEEE Transactions on Microwave Theory and Techniques, vol. 54, no. 1, pp. 278-284, 2006, https://doi.org/10.1109/TMTT.2005.860300.

[11] L. Wu, H. Yilmaz, T. Bitzer, and A. Berroth. "A dual-frequency Wilkinson power divider: for a frequency and its first harmonic", IEEE Microwave and Wireless Components Letters, vol. 15, no. 2, pp. 107-109, https://doi.org/10.1109.2004.842848.

[12] Rogers Corporation, " RO4000®Series High Frequency Circuit Materials, https://rogerscorp.com/-/media/project/rogerscorp /documents/advanced-connectivity-solutions/english/data -sheets /ro4000-laminates-ro4003c-and-ro4350b-data -sheet.pdf [27-Mar-2020].

[13] Cinch Connectivity Solutions Johnson, SMA 50 Ohm End Launch Jack Receptacle - Round Contact, https://www.digikey. com/htmldatasheets/production/21983/0/0/1/142-0701-881 -spec. html [29-June-2020]

Received 7 July 2020

Vaidotas Barzdenas was born in Vilnius, Lithuania, in 1980. He received the $\mathrm{BS}, \mathrm{MS}$, and $\mathrm{PhD}$ degrees in electronic and electrical engineering from Vilnius Gediminas Technical University, Vilnius, Lithuania, in 2002, 2004, and 2008, respectively. He is currently a Professor with the Department of Computer Science and Communications Technologies, Vilnius Gediminas Technical University. His current research interests include the novel micro- and nanoelectronics technologies, analog, mixed-signal, RF circuit and system design.

Aleksandr Vasjanov was born on July 26, 1989, in Klaipeda, Lithuania. He was awarded Bachelor's, Masters's and $\mathrm{PhD}$ degrees in electronics engineering at Vilnius Gediminas Technical University, Vilnius, Lithuania, in 2012, 2014, and 2019, respectively. His main fields of interest are radio frequency integrated circuit and system design, including power amplifier architecture, high-density RF/mixed signal printed circuit board design, as well as impedance matching network design and research.

Gediminas Grazulevicius was born in Vilnius, Lithuania, in 1973. He received the BS degree in mechanical engineering from the Vilnius Gediminas Technical University, Vilnius, Lithuania, in 1995, and the MS, and PhD degrees in electronic and electrical engineering from the Vilnius Gediminas Technical University in 2000 and 2004, respectively. Since 2004, he has been an Associate Professor with the Department of Computer Science and Communications Technologies, Vilnius Gediminas Technical University. His research interests include the electromagnetic disturbances, electromagnetic field measurement technologies, computer network security, and data mining.

Andzej Borel was born in was born in Vilnius, Lithuania, in 1994. He received BS and MS, in electronics and electrical engineering from Vilnius Gediminas Technical University, Vilnius, Lithuania in 2017 and 2019, respectively. He is currently pursing his $\mathrm{PhD}$ in Vilnius Gediminas Technical University. His current research interests include analog, mixed-signal, $\mathrm{RF}$ circuit and system design, particularly power amplifiers linearization techniques. 